\title{
OPTIMIZATION OF CUTTING PARAMETERS ON MILD STEEL WITH HSS \& CEMENTED CARBIDE TIPPED TOOLS USING ANN
}

\author{
A. V. N. L. Sharma ${ }^{1}$, P. Satyanarayana Raju ${ }^{2}$, A. Gopichand ${ }^{3}$, K. V. Subbaiah ${ }^{4}$ \\ ${ }^{1}$ Professor, ${ }^{2}$ Associate Professor, ${ }^{3}$ Professor, ${ }^{4}$ Professor \\ Avnls277522@gmail.com,psnraju8@gmail.com, allkagopichand@gmail.com
}

\begin{abstract}
Optimum Selection of cutting conditions importantly contribute to the increase of productivity and the reduction of cost, therefore utmost attention is paid to this problem in this contribution. In this paper, a neural network based approach to complex optimization of cutting parameters is proposed. To reach higher precision of the predicted results a neural optimization algorithm is developed and presented to ensure simple, fast and efficient optimization of all important turning parameters. The approach is suitable for fast determination of optimum cutting parameters during machining, where there is not enough time for deep analysis.
\end{abstract}

Surface roughness, an indicator of surface quality is one of the most specified customer requirements in a machining process. To predict the surface roughness, an Artificial Neural Network (ANN) model was designed through back propagation network using MATLAB 7.1 software for the data obtained.

Keywords: Surface Roughness, ANN, MATLAB.

\section{INTRODUCTION}

The important goal in the modern industries is to manufacture the products with lower cost and with high quality in short span of time. There are two main practical problems that engineers face in a manufacturing process. The first is to determine the values of process parameters that will yield the desired product quality (meet technical specifications) and the second is to maximize manufacturing system performance using the available resources.

Today, Metal cutting process places major portion of all the manufacturing processes. Within these metal cutting processes the turning operation is the most fundamental metal removal operation in the manufacturing industry. Surface roughness, which is used to determine and evaluate the quality of a product, and is one of the major quality attributes of a product obtained from turning operation. Surface roughness is a widely used as an index of product quality and in most cases a technical requirement for mechanical products $[1,2]$. Achieving the desired surface quality is of great importance for the functional behavior of a part. Surface roughness is a measure of the quality of a product and a factor that greatly influences manufacturing cost [3]. It can be generally stated that the lower the desired surface roughness the more the manufacturing cost and vice versa. In order to obtain better surface roughness, the proper setting of cutting parameters is crucial before the process takes place [4].

Factors such as spindle speed, feed rate, and depth of cut that control the cutting operation can be setup in advance. However, factors such as geometry of cutting tool, tool wear, and joint material properties of both tool and work piece are uncontrollable [5]. One should develop techniques to evaluate the surface roughness of a product before machining in order to determine the required machining parameters such as feed rate, spindle speed and depth of cut for obtaining a desired surface roughness and product quality $[6,7]$.

\section{EXPERIMENTAL PROCEDURE}

\section{Experimental details and specifications}

Machine tool : Engine Lathe

Work material : Mild steel

Cutting tool : High speed steel, Cemented carbide tipped tool Cutting conditions

: Dry environment

Surface roughness measuring instrument

Mitutoyo SJ-201P

Traverse Speed $: 1 \mathrm{~mm} / \mathrm{sec}$

Measurement : Metric/Inch

\begin{tabular}{|c|c|c|c|}
\hline Level & $\begin{array}{c}\text { Speed (v) } \\
\text { (rpm) }\end{array}$ & $\begin{array}{c}\text { Feed rate(f) } \\
(\mathbf{m m} / \mathbf{r e v})\end{array}$ & $\begin{array}{c}\text { Depth of cut(d) } \\
\text { (mm) }\end{array}$ \\
\hline 1 & 228 & 0.05 & 0.4 \\
\hline 2 & 450 & 0.08 & 0.6 \\
\hline 3 & 740 & 0.1 & 1 \\
\hline
\end{tabular}




\begin{tabular}{|c|c|c|c|c|c|c|c|c|c|c|}
\hline \multirow[t]{2}{*}{ S.No } & \multirow[t]{2}{*}{$\begin{array}{l}\text { Speed } \\
(\mathrm{rpm})\end{array}$} & \multirow{2}{*}{$\begin{array}{l}\text { Feed } \\
\text { (mm } \\
\text { /rev) }\end{array}$} & \multirow[t]{2}{*}{$\begin{array}{l}\text { Depth of } \\
\text { cut(mm) }\end{array}$} & \multirow{2}{*}{$\begin{array}{c}\text { Measured } \\
\text { Surface } \\
\text { roughness } \\
\operatorname{Ra}(\mu \mathrm{m})\end{array}$} & \multicolumn{6}{|c|}{$\begin{array}{c}\text { ANN Computed roughness Ra }(\mu \mathrm{m}) \\
\text { w.r.t hidden neurons }\end{array}$} \\
\hline & & & & & $\begin{array}{c}\text { @20 } \\
\text { neuro } \\
\text { ns }\end{array}$ & $\begin{array}{l}\text { Deviati } \\
\text { on (\%) }\end{array}$ & $\begin{array}{c}\text { @25 } \\
\text { neuro } \\
\text { ns }\end{array}$ & $\begin{array}{l}\text { Deviati } \\
\text { on (\%) }\end{array}$ & $\begin{array}{c}@ 30 \\
\text { neuro } \\
\text { ns }\end{array}$ & $\begin{array}{l}\text { Deviati } \\
\text { on (\%) }\end{array}$ \\
\hline 1 & 250 & 0.06 & 0.5 & 1.96 & 1.902 & -5.78 & 1.993 & 3.310 & 1.899 & -6.011 \\
\hline 2 & 250 & 0.06 & 0.7 & 2.10 & 2.162 & 6.221 & 1.914 & -18.51 & 1.879 & 20.21 \\
\hline 3 & 250 & 0.06 & 0.9 & 2.07 & 1.795 & -27.41 & 1.859 & -21.05 & 1.863 & -20.64 \\
\hline 4 & 360 & 0.07 & 0.5 & 1.84 & 2.013 & 17.32 & 1.851 & 1.122 & 1.899 & 5.941 \\
\hline 5 & 360 & 0.07 & 0.7 & 1.98 & 2.179 & 19.93 & 1.889 & -9.090 & 1.813 & -8.442 \\
\hline 6 & 360 & 0.07 & 0.9 & 2.09 & 2.354 & 26.48 & 2.178 & 8.832 & 1.787 & 30.29 \\
\hline 7 & 540 & 0.09 & 0.5 & 2.04 & 1.513 & -46.25 & 1.973 & -6.621 & 1.996 & -4.347 \\
\hline 8 & 540 & 0.09 & 0.7 & 2.10 & 2.083 & -1.970 & 2.233 & 13.31 & 2.297 & 19.73 \\
\hline 9 & 540 & 0.09 & 0.9 & 2.28 & 2.124 & -15.93 & 2.359 & 7.941 & 2.227 & -5.729 \\
\hline \multicolumn{5}{|c|}{ Average } & & 18.589 & & 9.782 & & $\begin{array}{l}13.48 \\
\end{array}$ \\
\hline
\end{tabular}

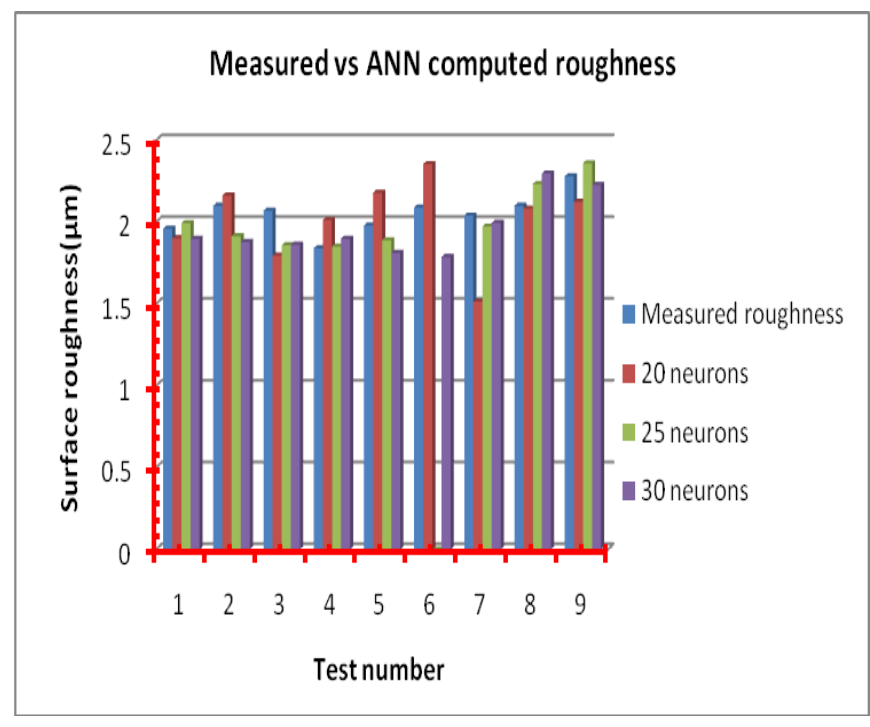

\begin{tabular}{|c|c|c|c|c|c|c|c|c|c|c|}
\hline \multirow[t]{2}{*}{ S.No } & \multirow[t]{2}{*}{$\begin{array}{l}\text { Speed } \\
(\mathrm{rpm})\end{array}$} & \multirow{2}{*}{$\begin{array}{l}\text { Feed } \\
\text { (mm } \\
/ \text { rev) }\end{array}$} & \multirow{2}{*}{$\begin{array}{l}\text { Depth } \\
\text { of cut } \\
\text { (mm) }\end{array}$} & \multirow{2}{*}{$\begin{array}{c}\text { Measured } \\
\text { Surface } \\
\text { Roughness } \\
\operatorname{Ra}(\mu \mathrm{m})\end{array}$} & \multicolumn{6}{|c|}{$\begin{array}{l}\text { ANN Computed roughness } \mathrm{Ra}(\mu \mathrm{m}) \\
\text { w.r.t hidden neurons }\end{array}$} \\
\hline & & & & & $\begin{array}{c}\text { @20 } \\
\text { neuro } \\
\text { ns }\end{array}$ & $\begin{array}{l}\text { Deviati } \\
\text { on (\%) }\end{array}$ & $\begin{array}{c}\text { @25 } \\
\text { neuro } \\
\text { ns }\end{array}$ & $\begin{array}{l}\text { Deviati } \\
\text { on (\%) }\end{array}$ & $\begin{array}{c}@ 30 \\
\text { neuro } \\
\text { ns }\end{array}$ & $\begin{array}{l}\text { Deviati } \\
\text { on (\%) }\end{array}$ \\
\hline 1 & 250 & 0.06 & 0.5 & 2.86 & 2,931 & 6.313 & 2.873 & 0.475 & 3.091 & 22,92 \\
\hline 2 & 250 & 0.06 & 0.7 & 2.86 & 3.058 & \begin{tabular}{|l|l|}
18.31 \\
\end{tabular} & 2.958 & 9.078 & 2.915 & 4.796 \\
\hline 3 & 250 & 0.06 & 0.9 & 2.85 & 2,661 & 19,36 & 2.953 & 9.935 & 2.909 & 5.514 \\
\hline 4 & 360 & 0.07 & 0.5 & 2.83 & 2.903 & 6.708 & 2.811 & -2.529 & 2.990 & 15.36 \\
\hline 5 & 360 & 0.07 & 0.7 & 2.88 & 2.936 & 5.518 & 2.746 & 13.47 & 2.893 & 1.212 \\
\hline 6 & 360 & 0.07 & 0.9 & 2.90 & 2.768 & $.13,33$ & 2.720 & -18.06 & 2.912 & 1.123 \\
\hline 7 & 540 & 0.09 & 0.5 & 3.14 & 3.151 & 0.740 & 3.048 & $-9,389$ & 2.968 & 17.97 \\
\hline 8 & 540 & 0.09 & 0.7 & 3.17 & 3.270 & 10.14 & 3.202 & 2.430 & 3.057 & $-12,07$ \\
\hline 9 & 540 & 0.09 & 0.9 & 3.19 & 3.287 & 8.774 & 3.189 & -1.014 & 3.212 & 1.357 \\
\hline \multicolumn{5}{|c|}{ Average } & & 9.900 & & 7.376 & & 9.147 \\
\hline
\end{tabular}

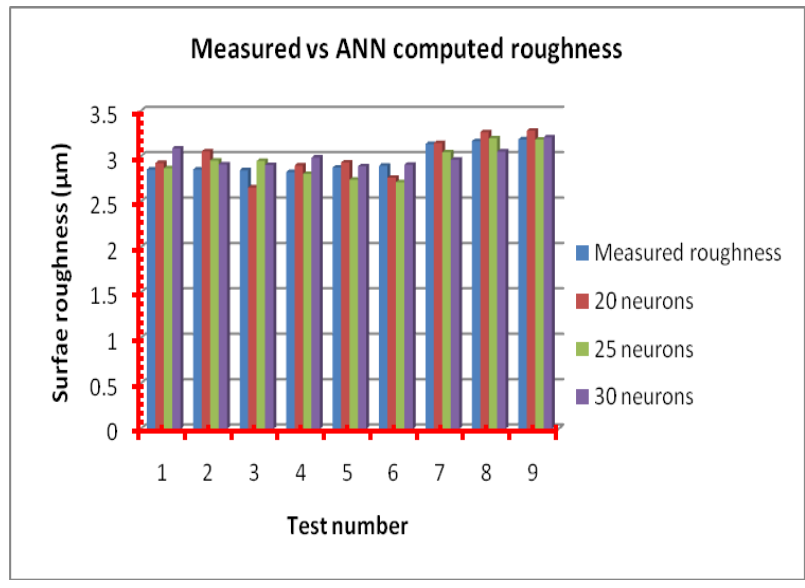

\section{HSS tool on mild steel}

\begin{tabular}{|c|c|c|c|c|c|c|}
\hline \multicolumn{3}{|c|}{ Training performance } & \multicolumn{3}{c|}{ Test performance } \\
\hline $\begin{array}{c}\text { Hidden } \\
\text { neurons }\end{array}$ & 20 & 25 & 30 & 20 & 25 & 30 \\
\hline Regression & 0.998 & 0.999 & 0.998 & 0.873 & 0.91 & 0.86 \\
\hline
\end{tabular}

\section{Cemented carbide tipped tool on mild steel}

\begin{tabular}{|c|c|c|c|c|c|c|}
\hline \multicolumn{3}{|c|}{ Training performance } & \multicolumn{3}{c|}{ Test performance } \\
\hline $\begin{array}{c}\text { Hidden } \\
\text { neurons }\end{array}$ & 20 & 25 & 30 & 20 & 25 & 30 \\
\hline Regression & 0.995 & 0.997 & 0.995 & 0.855 & 0.93 & 0.850 \\
\hline
\end{tabular}

\section{Optimum cutting Conditions:}

\begin{tabular}{|c|c|c|c|c|c|}
\hline \multirow{2}{*}{$\begin{array}{c}\text { Type of } \\
\text { tool } \\
\begin{array}{c}\text { Used on } \\
\text { MS }\end{array}\end{array}$} & $\begin{array}{c}\text { Speed } \\
(\mathrm{rpm})\end{array}$ & $\begin{array}{c}\text { Feed } \\
(\mathrm{mm} / \mathrm{rev})\end{array}$ & $\begin{array}{c}\text { Depth } \\
\text { of } \\
\mathrm{Cut} \\
(\mathrm{mm})\end{array}$ & $\begin{array}{c}\text { ANN } \\
\mathrm{Ra} \\
(\mu \mathrm{m})\end{array}$ & $\begin{array}{c}\% \\
\text { approach } \\
\text { by ANN } \\
\text { Technique }\end{array}$ \\
\hline HSS & 729 & 0.056 & 0.44 & 1.73 & 92 \\
\hline $\begin{array}{c}\text { Cemented } \\
\text { Carbide }\end{array}$ & 702 & 0.051 & 0.416 & 2.74 & 93 \\
\hline
\end{tabular}

The correlation coefficient (R) of test performance of HSS and cemented carbide tool on mild steel, is 0.91 and 0.93 and their $\mathrm{R}^{2}$ values are 0.821 and 0.864 respectively,

Which means that $82 \%$ and $86 \%$ of the total variation in network prediction can be explained by the linear relationship between experimental values and network predicted values

The other $18 \%$ and $14 \%$ of the total variation in network prediction remains unexplained.

The value of $\mathrm{R}^{2}$ increases up to hidden neuron 25 . Then it starts to decrease mainly in terms of testing cases. So, 
the network consisting of 25 hidden neurons was selected as the optimum one in this present work.

\section{REFERENCES}

[1] Adeel H. Suhail, N. Ismail, S.V. Wong and N.A. Abdul Jalil [2010], Optimization of Cutting Parameters Based on Surface Roughness and Assistance of Workpiece Surface Temperature in Turning Process, American J. of Engineering and Applied Sciences 3 (1): 102-108, 2010

[2] Adesta Erry Yulian T., Riza Muhammad, Hazza Muataz, Agusman Delvis, Rosehan, Tool Wear and Surface Finish Investigation in High Speed Turning Using Cermet Insert by Applying Negative Rake Angles, European Journal of Scientific Research Vol.38 No.2 (2009): pp.188.

[3] Ali, S.M. and Dhar, N.R., 2010, "Tool Wear and Surface Roughness Prediction using an artificial Neural Network in Turning Steel under Minimum Quantity Lubrication", International Conference on Industrial EngineeringICIE-2010, WASET Conference Proceedings, 62(4), 607-616.

[4] Aslan E, Camuşcu N, Bingören B (2007). Design optimization of cutting parameters when turning hardened AISI 4140 (63 HRC) with Al2O3+TiCN mixed ceramic tool, Mater. Design, 28: 1618-1622.

[5] A. Kohli · U.S. Dixit [2005], "A neural-network-based methodology for the prediction of surface roughness in a turning process" Int J Adv Manuf Technol (2005) 25: 118-129 Ishibuchi H, Tanaka H (1991) Regression analysis with interval model by neural networks. Proc of the IEEE Int Joint Conf on Neural Networks, Singapore, pp 1594-1599.

[6] Abburi NR, Dixit US (2006). A knowledge-based system for the prediction of surface roughness in turning process. Robotics and Comp.-Integ. Manu., 22: 363-372.

[7] Asilturk I, Cunkas M (2011). Modelling and prediction of surface roughness in turning operations using artificial neural network and multiple regression method. Expert Sys. Appl., 38(5): 5826-5832. 